\title{
Estado da produção científica internacional em Born Globals
}

O presente artigo tem por objetivo analisar o estado da arte da produção científica internacional sobre "born globals". Para isso, um procedimento metodológico bibliométrico foi adotado para o desenvolvimento da pesquisa, baseado em periódicos científicos internacionais presentes nas bases de dados Science Direct, Web of Science e EBSCO. Como objetivos específicos, pretende-se identificar os estudos mais relevantes sobre o tema, verificar qual a contribuição desses estudos para o avanço do conhecimento sobre a estratégias de internacionalização de empresas e identificar os autores, revistas e artigos mais relevantes para o tema.

Palavras-chave: Internacionalização; Organizações; Estratégia.

\section{State of international scientific production at Born Globals}

The aim of this article is to analyze the state of the art of scientific production on "born globals". For this, a methodological bibliometric method was adopted for the development of the research, to be downloaded in scientific and international journals in the databases Direct Science, Web of Science and EBSCO. As specific objectives, we intend to identify the most relevant studies on the subject, verify the quality of the internationalization of companies and identify the authors, journals and articles most relevant to the theme.

Keywords: Internationalization; Organizations; Strategy.

Topic: Planejamento, Estratégia e Competitividade

Reviewed anonymously in the process of blind peer.
Received: 10/10/2017

Approved: 10/12/2017
Issa Ibrahim Berchin

Universidade do Sul de Santa Catarina, Brasil

http://lattes.cnpq.br/6154133328702720

issaberchim@gmail.com

\section{Alexsandro da Silva}

Universidade do sul de Santa Catarina, Brasil

alexsandro.silva2@unisul.br

\section{Graciella Martignago}

Universidade do Sul de Santa Catarina, Brasil http://lattes.cnpq.br/2116155644461800

graciella.martignago@unisul.br

\author{
Wlamir Gonçalves Xavier \\ Universidade do Vale do Itajaí, Brasil \\ http://lattes.cnpq.br/6121935361338902 \\ wlamir.xavier@unisul.br
}

Ana Barbara Silveira Mendonça Santos Dias Universidade do Sul de Santa Catarina, Brasil http://lattes.cnpq.br/0275332281303252 anabarbaramendonca@ymail.com

José Baltazar Salgueirinho Osorio de Andrade Guerra

Universidade do Sul de Santa Catarina, Brasil

http://lattes.cnpq.br/1865825655857573

baltazar.guerra@unisul.br
Referencing this:

BERCHIN, I. I.; SILVA, A.; MARTIGNAGO, G.; XAVIER, W. G.; DIAS, A. B. S. M; S.; GUERRA, J. B. S. O. A.. Estado da produção científica internacional em Born Globals. Revista Brasileira de Administração Científica, v.8, n.3, p.85-98, 2017. DOI:

http://doi.org/10.6008/SPC2179-684X.2017.003.0007 


\section{INTRODUÇÃO}

No estudo das teorias clássicas de internacionalização de empresas, duas perspectivas são destacadas, uma comportamental e outra econômica (ANDERSEN et al., 2002; NEUMANN et al., 2005). Andersen et al. (2002) destaca que a abordagem econômica considera que as decisões tomadas acerca do processo de internacionalização são predominantemente racionais e objetivas. A abordagem econômica é bastante útil para analisar o desenvolvimento posterior da internacionalização, ou seja, momentos em que a firma já se encontra em estágios mais avançados e maduros do processo de internacionalização (ANDERSEN et al., 2002). Nesta corrente teórica destacam-se as teorias do comércio internacional, do ciclo de vida e teoria neoclássica de localização (WEISFELDER, 2001; IETTO-GILLIES, 2002).

No que se refere às teorias econômicas, estas destacam-se através das teorias tradicionais, que com a contribuição dos diferentes autores desenvolveram modelos assentados na vantagem comparativa (NORDSTRÖM et al., 1992). Quanto às teorias comportamentais, originaram-se dos chamados 'modelos de estágios', com o modelo de internacionalização de Uppsala (JOHANSON et al., 1990; JOHANSON et al., 1975). Posteriormente, surgiu a teoria das redes (JOHANSON et al., 1988), e a partir da década de 1990, as teorias de empreendedorismo internacional (JOHANSON et al., 1988) e das born globals (RENNIE, 1993). Estas teorias mais recentes procuram analisar o fenômeno específico que é a internacionalização acelerada de pequenas empresas.

O Modelo de Uppsala foi desenvolvido a partir de estudos de casos sobre a internacionalização de empresas suecas. Esses estudos mostravam que essas empresas desenvolviam suas operações internacionais em pequenos passos, em vez de fazer grandes investimentos no exterior de uma só vez. Devido a contribuições de outros autores escandinavos à perspectiva proposta pelos teóricos de Uppsala, este modelo é também conhecido como da Escola Nórdica (WEISFELDER, 2001; HEMAIS et al., 2004).

Os autores da Escola Nórdica procuraram entender o processo de internacionalização como um processo gradual, sendo resultado de decisões incrementais. Johanson et al. (1975) propusera, então, a existência de uma 'cadeia de estabelecimento'. Segundo a cadeia constituída de quatro estágios, o processo de internacionalização apresenta crescente comprometimento de recursos ao longo do seu desenvolvimento. O Modelo de Uppsala preocupa-se em observar o processo de aquisição e desenvolvimento da experiência e do conhecimento sobre o mercado internacional (JOHANSON et al., 1975).

Esse aprendizado é a base do compromisso crescente e alocação de recursos no mercado estrangeiro. Em outras palavras, o nível de comprometimento dos recursos da empresa aumenta à medida que ela ganha conhecimento e experiência sobre o mercado em questão. Para Wiedersheim-Paul et al. (1978), existiriam diferentes fatores internos e externos à empresa que chamariam a atenção dos tomadores de decisão. O tipo e o tempo de atenção dedicada a esses fatores dependeriam, então, das características do próprio tomador de opinião, do ambiente externo e interno da empresa.

O grau de comprometimento dos recursos disponibilizados pela empresa, cresce à medida que se conhece o mercado, constituindo um ciclo: o crescente conhecimento do mercado facilitaria a percepção de ameaças ou oportunidades, possibilitando a tomada de decisão a respeito da internacionalização. Assim, a 
medida que se compromete os recursos, aumenta o conhecimento do mercado, possibilitando a verificação das ameaças e oportunidades (TANURE et al., 2007).

Johanson et al. (2009) destaca que, quando uma empresa toma a decisão de expandir os seus mercados para um nível internacional, deve considerar a configuração da rede a qual essa faz parte, pois isto influencia na sua entrada em outros países. Alguns dos motivos que levam as organizações a optarem pela internacionalização passam pelo facto do mercado interno estar saturado e haver a oportunidade de aquando a presença num determinado país seja concedido o acesso a recursos estratégicos ou então existirem efeitos clusters a serem explorados numa determinada região.

Teixeira et al. (2005) destaca as motivações para a internacionalização são apresentadas a partir de seis fatores, como o acesso a recurso mais baratos e 'melhores', o maior retorno do investimento, o aumento da quota de mercado, a fuga à importação, ou demanda de importação, como a resposta a clientes/concorrentes e o acesso a competências.

Outro modelo de internalização na abordagem comportamental é o modelo de Born Globals, onde sua evolução de internacionalização se dá por fases. Desde sua criação, as Born Globais, são criadas com a elaboração de estratégias internacionais, criando imediatamente no exterior após sua de subsidiárias (OVIATT et al., 1994). Em síntese, são várias as propostas dos autores quanto às motivações para a internacionalização, estando estas relacionadas a fatores internos ou externos à empresa e derivando da fase do processo de expansão internacional em que esta se encontra.

\section{Born Globals}

O conceito de Born Global foi introduzido pela primeira vez na literatura por Rennie (1993), como um termo padrão para rápida internacionalização de pequenas e médias empresas, e tem sido definido de muitas outras maneiras desde então. Granitsky (1989) foi um dos primeiros a citar uma empresa deste tipo, em seu estudo de 18 empresas exportadoras Israelitas, o qual chamou-as de empresas exportadoras inatas.

Alguns anos mais tarde, Jolly et al. (1992) estava investigando empresas de alta tecnologia e classificou-as de empresas internacionais de criação de alta tecnologia, Startups. Em 1993, Rennie (1993) investigou 310 exportadores australianos e os classificou dois tipos de empresas, as exportadoras tradicionais e Born Globals. Esta foi a primeira vez que o termo Born Global foi utilizado, e Rennie (1993) os definiu como grupo de empresas que normalmente exportam $75 \%$ das suas vendas e iniciou a exportação nos primeiros 2 anos de tempo de vida da empresa.

Oviatt et al. (1994) relata que o processo de rápida internacionalização de uma empresa pode traduzir-se também no conceito de 'International New Ventures', tratando-se de organizações empresariais que, desde o início, procuram obter vantagem competitiva significativa, através do uso dos recursos e das vendas pelos vários países. Para Knight et al. (1996), os fatores que levam à existência de Born Globals estão relacionados com o número crescente de nichos de mercado presentes no mundo, nas mudanças na tecnologia de produção, comunicação e no número crescente de redes internacionais. 
Ao contrário de outras, as empresas Born Globals não tem seu crescimento de forma gradual, pois são criadas para o mercado internacional e veem o mercado interno como um apoio para os seus negócios (RENNIE, 1993). As Born Globals têm por objetivo alcançar mercados internacionais, ou até mesmo globais, desde sua fundação ou rapidamente após esta (MADSEN et al., 1997), sem se dedicarem, em primeiro lugar, a atividades no mercado doméstico (GABRIELSSON, 2005).

Em comparação com outras empresas exportadoras, Born Globals estão focadas em estratégias de nicho, utilizando tecnologia de ponta para desenvolver produtos altamente especializados (bens ou serviços) apropriados para nichos de mercado globais (BARONCHELLI et al., 2014). Portanto, essas empresas tendem a fornecer produtos com alto valor agregado para os mercados internacionais (KNIGHT et al., 2004). Para Forrest (1990), o desenvolvimento de produtos e processos inovadores é a arma competitiva destas empresas. Embora reconhecendo essas oportunidades, as Born Globals enfrentam muitos desafios na concorrência internacional (KARAGOZOGLU et al., 1998; MOEN, 2002; NORDMAN et al., 2008).

De acordo com Weerawardena (2007), as Borns Globals são relativamente vulneráveis em comparação com as grandes multinacionais, pois elas têm menos recursos a serem dirigidos ao esforço de internacionalização e de alta flutuações do mercado. Knight et al. (2004) sugere que, embora a maioria das Born Global não tenham recursos tangíveis substanciais, eles possuem um outro conjunto recursos fundamentais, composta dos intangíveis, que facilitam o sucesso internacional. Para Freeman (2013), o empresário é fundamental para o processo de internacionalização destas empresas.

Harveston et al. (2000) e Zhang et al. (2013) também conclui que as atitudes e a mentalidade da equipe de gestão desempenham um papel importante no nível de envolvimento em atividades internacionais. Segundo Harveston et al. (2000) e Zhang et al. (2013), Born Globals possuem uma mentalidade global e os seus gestores têm maior experiência internacional e estão mais dispostas para aceitar o risco.

Dib et al. (2010) afirma que os empresários de Born Globais, parecem ser mais sensíveis aos riscos associados com operações em mercados internacionais do que empresas que adotam um caminho de internacionalização gradual. Neste sentido, gestão de riscos e adaptação são fundamentais para a dinâmica dessas empresas, respondendo à inovação, mudança rápida e constante expansão do mercado.

Também é importante destacar que Born Globals recorrerem com mais frequência ao uso de redes pessoais e empresariais para superar suas restrições (FREEMAN, 2012) e para atingir rapidamente um alcance global (RIALP-CRIADO, 2005). De acordo com Zhang et al. (2009), Born Globals têm uma maior capacidade de aprendizagem internacional para operar em redes e maior experiência internacional do que as empresas exportadoras tradicionais. Em contraste com as empresas multinacionais que se internacionalizam gradualmente, estas novas empresas se inserem no mercado com uma estratégia internacional proativa.

\section{METODOLOGIA}

A presente pesquisa possui natureza básica, por contribuir para a ciência através da criação de novos conhecimentos, mas sem aplicação prática (PRODANOV et al., 2013), adota abordagem qualitativa por estar 
relacionada às áreas sociais, através de análise subjetiva dos dados e do envolvimento direto do pesquisador (ASSIS, 2014; ERLINGSSON et al., 2013; APPOLINÁRIO, 2009).

A pesquisa também tem caráter exploratório, por ampliar o conhecimento dentro de uma temática (ASSIS, 2014), de acordo com procedimento bibliográfico e documental, através da análise de artigos científicos, conferência internacionais e relatórios de organismos internacionais (SILVA, 2004), para elaboração da fundamentação teórica apresentada na introdução.

Além disto, este artigo é uma revisão sistemática de literatura envolvendo a utilização de dados primários e secundários. Uma revisão sistemática de literatura pode ser considerada como uma revisão que visa responder a uma questão específica através do estudo proposto e emprega procedimentos metódicos e claros para responder à questão levantada (ASPFORS et al., 2015; MARCONI et al., 2003; CASTRO, 2001).

\section{Análise Bibliométrica}

Para o desenvolvimento desta análise, primeiro os autores identificaram o tema a ser analisado, em seguida selecionaram a palavra-chave Born Global para fundamentar a pesquisa, após isso, elaboraram a pergunta norteadora desta pesquisa (terceira etapa). A quarta etapa foi a seleção das bases de dados a serem utilizadas para esta pesquisa.

As bases de dados selecionadas foram a Web of Science, a Science Direct e a EBSCO. Essas bases de dados foram escolhidas pela sua relevância para o meio acadêmico. A base de dados Web of Science apoia 15 bases de dados diferentes, com um número aproximado de publicações de 2,6 milhões de documentos (TR, 2015). Por sua vez, a Science Direct, apresenta 1/4 dos conteúdos científicos e técnicos do mundo e têm quase 2.500 periódicos, mais de 30.000 livros e um valor aproximado de 13 milhões e 400 mil artigos, baseado em dados da ELSEVIER. Já a EBSCO, ou Business Source Complete, conta com mais de 1.200 periódicos focados em disciplinas relacionadas a negócios, incluindo marketing, administração, MIS, POM, contabilidade, finanças e economia (UTL, 2015).

Após a definição do tema, da palavra-chave, da pergunta e das bases de dados, a quinta etapa referese à delimitação dos filtros de exclusão, baseados na presença da palavra-chave Born Global no título dos artigos, publicados em revistas científicas das áreas de administração, negócios, contabilidade e empreendedorismo.

Então, após a seleção do tema, elaboração da pergunta de pesquisa, definição dos filtros de exclusão e seleção das bases de dados, os filtros foram aplicados e, a partir da leitura dos títulos dos artigos filtrados, os autores pré-selecionaram os artigos mais relevantes de cada base de dados (sexta etapa). Consideraram os artigos mais relevantes aqueles cujos títulos são relacionados com estratégias de internacionalização de empresas.

A partir disso, os autores iniciaram a próxima etapa desta pesquisa, referente a leitura dos resumos e seleção final dos artigos a serem examinados (sétima etapa). A leitura dos resumos permitiu que 44 artigos fossem selecionados (oitava etapa). A tabela 1 ilustra o processo sequencial de seleção dos artigos mais 
pertinentes para esta pesquisa, mostrando os números absolutos de artigos em cada base de dados, de acordo com a aplicação dos filtros.

Tabela 1: Processo sequencial de seleção dos artigos mais pertinentes para Born Globals.

\begin{tabular}{|l|l|l|l|}
\hline Aplicação sequencial dos filtros & Science Direct & Web of Science & EBSCO \\
\hline Total de artigos nas bases de dados & 85.377 & 11.752 & 318 \\
\hline Total de artigos (palavra-chave no título) & 3 & 102 & 82 \\
\hline Filtro por tipo de publicação (artigo científico) & 3 & 67 & 73 \\
\hline Filtro por língua (inglês) & 2 & 47 & 66 \\
\hline Filtro por área: administração, contabilidade e negócios & 2 & 45 & 55 \\
\hline Filtro pela percepção dos autores: Leitura dos títulos & 2 & 24 & 26 \\
\hline Filtro pela percepção dos autores: Leitura dos resumos & 2 & 23 & 19 \\
\hline
\end{tabular}

A nona etapa consiste na exclusão de artigos duplicados. Após a seleção final dos artigos e agrupamento das 3 bases de dados em um único arquivo, os autores excluíram 14 artigos duplicados, restando apenas 30 artigos para a análise. Adicionalmente, artigos que publicações sem fator de impacto, ou sem classificação pela Capes, foram excluídos (décima etapa). Com o objetivo de simplificar a visualização holística do processo bibliométrico adotado nesta pesquisa os autores elaboraram a figura 1.

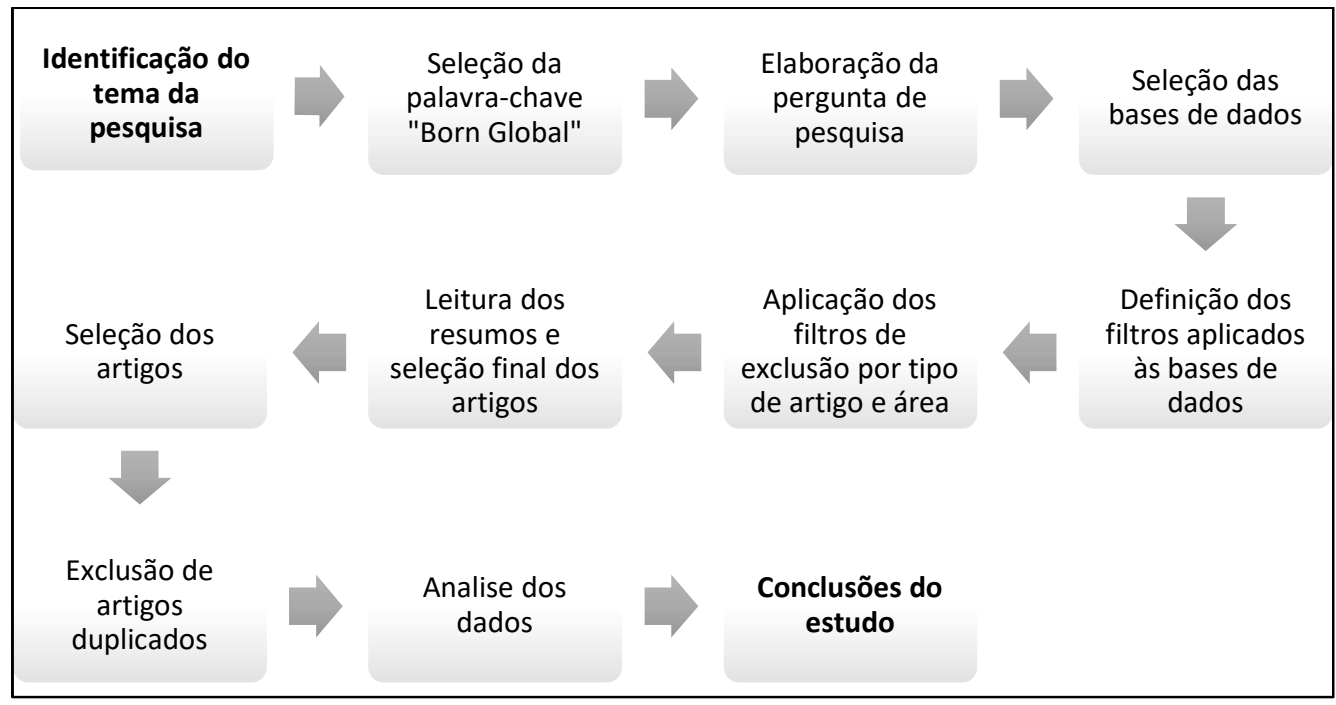

Figura 1: Etapas de desenvolvimento dos procedimentos bibliométricos desta pesquisa.

Os artigos selecionados são considerados os mais relevantes para esta pesquisa. Tendo isso em vista, as próximas seções apresentarão as etapas 10 e 11, referentes a análise e considerações finais desta pesquisa, respectivamente. Vale ressaltar que os autores se basearam nos artigos oriundos do bibliométrico para fundamentar a revisão de literatura.

\section{REVISÃO TEÓRICA}

A partir do processo bibliométrico aplicado a esta pesquisa, os autores puderam selecionar um conjunto de artigos que trata da internacionalização de empresas, mais especificamente, de Born Globals. A figura 2 representa o número de artigos publicados, levantados através do procedimento bibliométrico previamente descrito. Com o objetivo de melhor expor os artigos selecionados, a tabela 2 apresenta o conjunto de artigos científicos identificados, incluindo o nome dos autores, o título do artigo, o ano de publicação e a revista onde foi publicado. 


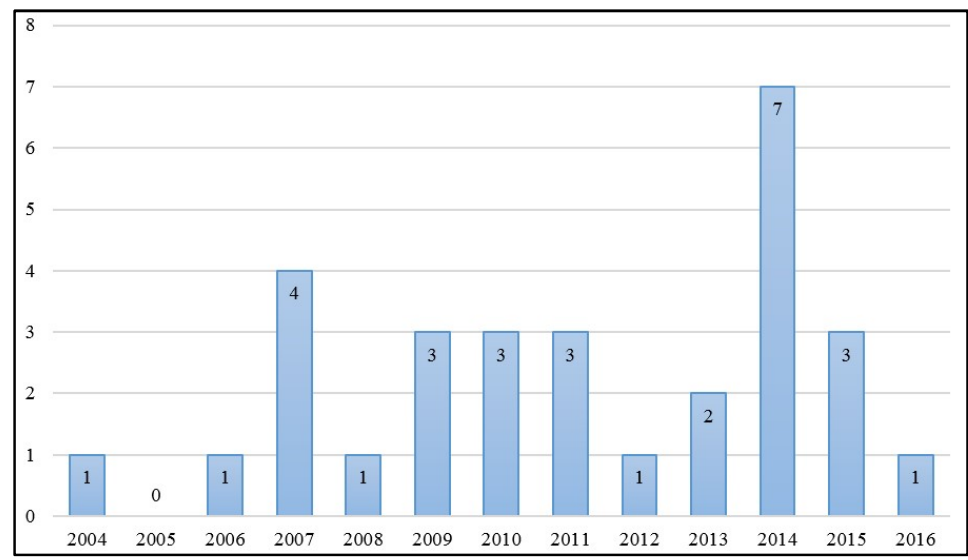

Figura 2: Evolução da produção científica sobre Born Globals.

Tabela 2: Artigos selecionados.

\begin{tabular}{|c|c|c|c|}
\hline Autor(es) & Ano & Título & Revista \\
\hline Knight \& Liesch & 2016 & Internationalization: From incremental to born global & Journal Of World Business \\
\hline $\begin{array}{l}\text { Monferrer, Blesa \& } \\
\text { Ripollés }\end{array}$ & 2015 & $\begin{array}{l}\text { Born globals trough knowledge-based dynamic capabilities and network market } \\
\text { orientation }\end{array}$ & $\begin{array}{l}\text { Brq Business Research } \\
\text { Quarterly }\end{array}$ \\
\hline Torres-Ortega & 2015 & How to measure born-global firms' orientation towards international markets? & $\begin{array}{l}\text { Revista Española De } \\
\text { Investigación En } \\
\text { Marketing ESIC }\end{array}$ \\
\hline $\begin{array}{l}\text { Gerschewski, Rose \& } \\
\text { Lindsay }\end{array}$ & 2015 & $\begin{array}{l}\text { Understanding the drivers of international performance for born global firms: An } \\
\text { integrated perspective }\end{array}$ & Journal Of World Business \\
\hline Cancino & 2014 & Rapid Internationalization of SMEs: Evidence from Born Global Firms in Chile & Innovar \\
\hline $\begin{array}{l}\text { Almor, Tarba \& } \\
\text { Margalit }\end{array}$ & 2014 & $\begin{array}{l}\text { Maturing, Technology-Based, Born-Global Companies: Surviving Through } \\
\text { Mergers and Acquisitions }\end{array}$ & $\begin{array}{l}\text { Management } \\
\text { International Review }\end{array}$ \\
\hline Hagen \& Zucchella & 2014 & Born Global or Born to Run? The Long-Term Growth of Born Global Firms & $\begin{array}{l}\text { Management } \\
\text { International Review }\end{array}$ \\
\hline Trudgen \& Freeman & 2014 & $\begin{array}{l}\text { Measuring the Performance of Born-Global Firms Throughout Their } \\
\text { Development Process: The Roles of Initial Market Selection and } \\
\text { Internationalisation Speed }\end{array}$ & $\begin{array}{l}\text { Management } \\
\text { International Review }\end{array}$ \\
\hline Baronchelli \& Cassia & 2014 & Exploring the antecedents of born-global companies' international development & $\begin{array}{l}\text { International } \\
\text { Entrepreneurship And } \\
\text { Management Journal }\end{array}$ \\
\hline Kaur \& Sandhu & 2014 & Internationalisation of born global firms: Evidence from Malaysia & $\begin{array}{l}\text { Journal Of The Asia Pacific } \\
\text { Economy }\end{array}$ \\
\hline Nummela & 2014 & $\begin{array}{l}\text { Strategic Decision-Making of a Born Global: A Comparative Study From Three } \\
\text { Small Open Economies }\end{array}$ & $\begin{array}{l}\text { Management } \\
\text { International Review }\end{array}$ \\
\hline Madsen & 2013 & $\begin{array}{l}\text { Early and rapidly internationalizing ventures: Similarities and differences } \\
\text { between classifications based on the original international new venture and } \\
\text { born global literatures. }\end{array}$ & $\begin{array}{l}\text { Journal Of International } \\
\text { Entrepreneurship }\end{array}$ \\
\hline Taylor \& Jack & 2013 & $\begin{array}{l}\text { Understanding the pace, scale and pattern of firm internationalization: An } \\
\text { extension of the 'born global' concept }\end{array}$ & $\begin{array}{l}\text { International Small } \\
\text { Business Journal }\end{array}$ \\
\hline Efrat \& Shoham & 2012 & $\begin{array}{l}\text { Born global firms: The differences between their short- and long-term } \\
\text { performance drivers }\end{array}$ & Journal Of World Business \\
\hline Hashai & 2011 & $\begin{array}{l}\text { Sequencing the expansion of geographic scope and foreign operations by "born } \\
\text { global" firms }\end{array}$ & $\begin{array}{l}\text { Journal Of International } \\
\text { Business Studies }\end{array}$ \\
\hline Efrat \& Shoham & 2011 & $\begin{array}{l}\text { Environmental characteristics and technological capabilities' interaction in high- } \\
\text { technology born global firms }\end{array}$ & $\begin{array}{l}\text { European Journal Of } \\
\text { International } \\
\text { Management }\end{array}$ \\
\hline Casas \& Vilma & 2011 & $\begin{array}{l}\text { The Impact Of External Business Environment Factors To Internationalization Of } \\
\text { "Born Global" Companies By Promoting Entrepreneurship }\end{array}$ & $\begin{array}{l}\text { Transformations In } \\
\text { Business \& Economics }\end{array}$ \\
\hline $\begin{array}{l}\text { Rialp-Criado, Galvan- } \\
\text { Sanchez \& Suarez- } \\
\text { Ortega }\end{array}$ & 2010 & $\begin{array}{l}\text { A configuration-holistic approach to born-global firms' strategy formation } \\
\text { process }\end{array}$ & $\begin{array}{l}\text { European Management } \\
\text { Journal }\end{array}$ \\
\hline Freeman & 2010 & A model of rapid knowledge development: The smaller born-global firm & $\begin{array}{l}\text { International Business } \\
\text { Review }\end{array}$ \\
\hline Dib, Rocha \& Silva & 2010 & $\begin{array}{l}\text { The internationalization process of Brazilian software firms and the born global } \\
\text { phenomenon: Examining firm, network, and entrepreneur variables. }\end{array}$ & $\begin{array}{l}\text { Journal Of International } \\
\text { Entrepreneurship }\end{array}$ \\
\hline $\begin{array}{l}\text { Zhang, Tansuhaj \& } \\
\text { McCullough }\end{array}$ & 2009 & $\begin{array}{l}\text { International entrepreneurial capability: The measurement and a comparison } \\
\text { between born global firms and traditional exporters in China. }\end{array}$ & $\begin{array}{l}\text { Journal Of International } \\
\text { Entrepreneurship }\end{array}$ \\
\hline $\begin{array}{l}\text { Holtbrugge \& } \\
\text { Wessely }\end{array}$ & 2009 & Initiating forces and success factors of born global firms & $\begin{array}{l}\text { European Journal Of } \\
\text { International } \\
\text { Management }\end{array}$ \\
\hline Crick & 2009 & The internationalisation of born global and international new venture SMEs & $\begin{array}{l}\text { International Marketing } \\
\text { Review }\end{array}$ \\
\hline
\end{tabular}




\begin{tabular}{|l|l|l|l|} 
Thai \& Chong & 2008 & Born-global: The case of four Vietnamese SMEs & $\begin{array}{l}\text { Journal Of International } \\
\text { Entrepreneurship }\end{array}$ \\
\hline Fan \& Phan & 2007 & $\begin{array}{l}\text { International new ventures: revisiting the influences behind the 'born-global' } \\
\text { firm }\end{array}$ & $\begin{array}{l}\text { Journal Of International } \\
\text { Business Studies }\end{array}$ \\
\hline $\begin{array}{l}\text { Laanti, Gabrielsson \& } \\
\text { Gabrielsson }\end{array}$ & 2007 & $\begin{array}{l}\text { The globalization strategies of business-to-business born global firms in the } \\
\text { wireless technology industry }\end{array}$ & $\begin{array}{l}\text { Industrial Marketing } \\
\text { Management }\end{array}$ \\
\hline Weerawardena & 2007 & $\begin{array}{l}\text { Conceptualizing accelerated internationalization in the born global firm: A } \\
\text { dynamic capabilities perspective }\end{array}$ & Journal Of World Business \\
\hline Freeman \& Cavusgil & 2007 & $\begin{array}{l}\text { Toward a typology of commitment states among managers of born-global firms: } \\
\text { A study of accelerated internationalization }\end{array}$ & $\begin{array}{l}\text { Journal Of International } \\
\text { Marketing }\end{array}$ \\
\hline $\begin{array}{l}\text { Freeman, Edwards \& } \\
\text { Schroder }\end{array}$ & 2006 & $\begin{array}{l}\text { How smaller born-global firms use networks and alliances to overcome } \\
\text { constraints to rapid internationalization }\end{array}$ & $\begin{array}{l}\text { Journal Of International } \\
\text { Marketing }\end{array}$ \\
\hline Knight \& Cavusgil & 2004 & Innovation, organizational capabilities, and the born-global firm & $\begin{array}{l}\text { Journal Of International } \\
\text { Business Studies }\end{array}$ \\
\hline
\end{tabular}

Um dos propósitos deste estudo é identificar os artigos mais relevantes, para tanto, a quantidade de citações do conjunto de artigos identificados foi levantada com base no Google Scholar Citation. Tendo isso em vista, a tabela 3 apresenta a quantidade de citações do conjunto de artigos selecionados. Esta tabela foi intitulada 'Quantidade de citações por artigo'.

Tabela 3: Quantidade de citações por artigo.

\begin{tabular}{|c|c|c|}
\hline Título & Citações & $\%$ \\
\hline Innovation, organizational capabilities, and the born-global firm & 1756 & 46 \\
\hline Conceptualizing accelerated internationalization in the born global firm: A dynamic capabilities perspective & 432 & 11,23 \\
\hline How smaller born-global firms use networks and alliances to overcome constraints to rapid internationalization & 321 & 8,41 \\
\hline Toward a typology of commitment states among managers of born-global firms: A study of accelerated internationalization & 210 & 5,5 \\
\hline International new ventures: revisiting the influences behind the 'born-global' firm & 183 & 4,79 \\
\hline The globalization strategies of business-to-business born global firms in the wireless technology industry & 155 & 4,06 \\
\hline A model of rapid knowledge development: The smaller born-global firm & 136 & 3,56 \\
\hline The internationalisation of born global and international new venture SMEs & 114 & 2,99 \\
\hline $\begin{array}{l}\text { The internationalization process of Brazilian software firms and the born global phenomenon: Examining firm, network, } \\
\text { and entrepreneur variables. }\end{array}$ & 94 & 2,46 \\
\hline $\begin{array}{l}\text { International entrepreneurial capability: The measurement and a comparison between born global firms and traditional } \\
\text { exporters in China. }\end{array}$ & 79 & 2,07 \\
\hline Born-global: The case of four Vietnamese SMEs & 79 & 2,07 \\
\hline Sequencing the expansion of geographic scope and foreign operations by "born global" firms & 55 & 1,44 \\
\hline A configuration-holistic approach to born-global firms' strategy formation process & 53 & 1,39 \\
\hline $\begin{array}{l}\text { Early and rapidly internationalizing ventures: Similarities and differences between classifications based on the original } \\
\text { international new venture and born global literatures. }\end{array}$ & 34 & 0,89 \\
\hline Understanding the pace, scale and pattern of firm internationalization: An extension of the 'born global' concept & 34 & 0,89 \\
\hline Born global firms: The differences between their short- and long-term performance drivers & 32 & 0,84 \\
\hline $\begin{array}{l}\text { Measuring the Performance of Born-Global Firms Throughout Their Development Process: The Roles of Initial Market } \\
\text { Selection and Internationalisation Speed }\end{array}$ & 17 & 0,44 \\
\hline Internationalisation of born global firms: Evidence from Malaysia & 13 & 0,34 \\
\hline Born Global or Born to Run? The Long-Term Growth of Born Global Firms & 12 & 0,31 \\
\hline Exploring the antecedents of born-global companies' international development & 12 & 0,31 \\
\hline Initiating forces and success factors of born global firms & 11 & 0,29 \\
\hline Strategic Decision-Making of a Born Global: A Comparative Study From Three Small Open Economies & 9 & 0,23 \\
\hline Maturing, Technology-Based, Born-Global Companies: Surviving Through Mergers and Acquisitions & 8 & 0,21 \\
\hline Understanding the drivers of international performance for born global firms: An integrated perspective & 7 & 0,18 \\
\hline Environmental characteristics and technological capabilities' interaction in high-technology born global firms & 6 & 0,16 \\
\hline Internationalization: From incremental to born global & 6 & 0,16 \\
\hline Born globals trough knowledge-based dynamic capabilities and network market orientation & 3 & 0,08 \\
\hline $\begin{array}{l}\text { The Impact Of External Business Environment Factors To Internationalization Of "Born Global" Companies By Promoting } \\
\text { Entrepreneurship }\end{array}$ & 1 & 0,03 \\
\hline How to measure born-global firms' orientation towards international markets? & - & - \\
\hline Rapid Internationalization of SMEs: Evidence from Born Global Firms in Chile & - & - \\
\hline Total & 3817 & $100 \%$ \\
\hline
\end{tabular}

Por ordem de relevância, a média de citação dos artigos publicados sobre o tema é aproximadamente 127 citações por artigo. $O$ artigo mais citado (Innovation, organizational capabilities, and the born-global firm) representa $46 \%$ das citações, sendo uma referência nos estudos de Born Globals. Além disso, os 5 artigos mais citados representam mais de 75\% das citações. Dos artigos identificados, 2 não possuem 
nenhuma citação. Considerando que as palavras-chave representam os temas mais importantes de um artigo, a figura 3 representa as palavras-chave mais citadas pelos artigos selecionados.

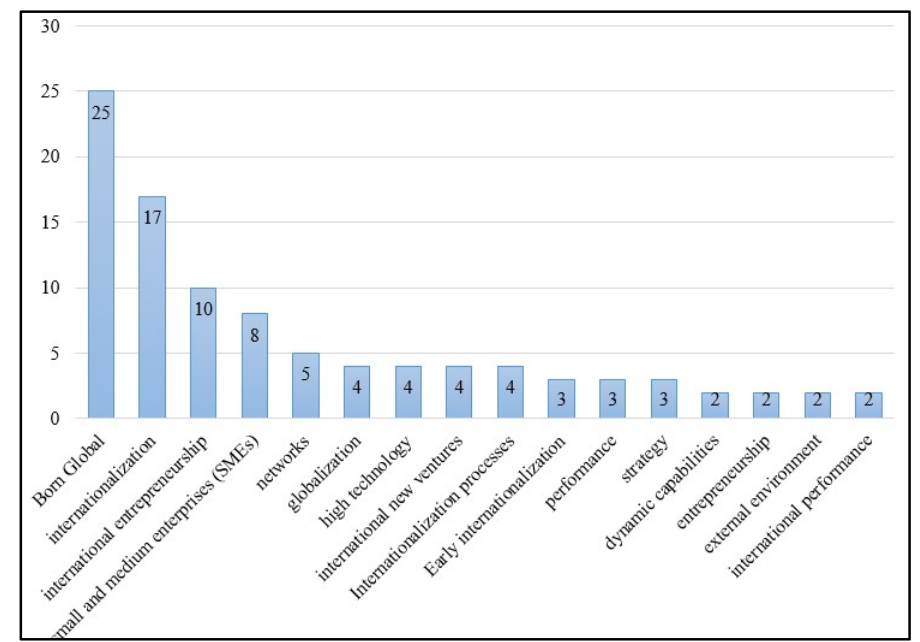

Figura 3: Palavras-chave mais citadas pelos artigos selecionados $(n=30)$.

A palavra-chave mais citada pelos 30 artigos selecionados foi Born Global, seguida por Internationalization, International Enterpreneurship e Small and medium enterprises (SMEs). O Gráfico 2 abordou apenas as palavras-chave que se repetiram. A presença da palavra-chave Born Global na maioria dos artigos é justificável pelo tema desta pesquisa, no entanto é interessante observar a presença das palavras-chave internacionalização, empreendedorismo, pequenas e médias empresas, network, globalização e tecnologias de ponta, palavras que vêm a caracterizar as empresas Born Globals. Tendo em vista a origem de publicação dos artigos, a tabela 4 apresenta as principais revistas científicas onde os artigos foram publicados.

Tabela 4: Revistas que mais publicaram artigos sobre o tema.

\begin{tabular}{|l|l|l|l|l|}
\hline \multicolumn{1}{|c|}{ Revista } & Fator de Impacto & \multicolumn{1}{c|}{$\begin{array}{c}\text { Classificação } \\
\text { WebQualis }\end{array}$} & Número de artigos & \% \\
\hline Journal of World Business & 2,388 & - & 4 & 13,33 \\
\hline Management International Review & 1,118 & $\mathrm{~A} 2$ & 4 & 13,33 \\
\hline Journal of International Entrepreneurship & - & - & 4 & 13,33 \\
\hline Journal of International Business Studies & 3,563 & $\mathrm{~A} 1$ & 3 & 10 \\
\hline Journal of International Marketing & - & - & 2 & 6,67 \\
\hline European Journal of International Management & - & - & 2 & 6,67 \\
\hline Outras & - & - & 11 & 36,67 \\
\hline
\end{tabular}

Observando a tabela 4 é possível perceber que 6, das 17 revistas de publicação dos artigos selecionados, acumulam cerca de 63,33\% das publicações dos artigos selecionados, com um total de 19 artigos, mostrando sua relevância na área de internacionalização de empresas, favorecendo o tema 'Born Global'. A tabela 5 apresenta a origem dos autores.

Tabela 5: Países que mais publicam sobre o tema.

\begin{tabular}{|l|l|l|}
\hline \multicolumn{1}{|c|}{ País de filiação dos autores por artigo } & Quantidade de autor & $\%$ \\
\hline Austrália & 13 & 20,31 \\
\hline Espanha & 8 & 12,5 \\
\hline Estados Unidos da América & 6 & 9,37 \\
\hline Finlândia & 6 & 9,37 \\
\hline Israel & 5 & 7,81 \\
\hline Itália & 4 & 6,25 \\
\hline
\end{tabular}




\begin{tabular}{|l|l|l|}
\hline Reino Unido da Grã-Bretanha & 4 & 6,25 \\
\hline Lituânia & 3 & 4,69 \\
\hline Brasil & 3,69 \\
\hline Alemanha & 3 & 3,12 \\
\hline Malásia & 2 & 3,12 \\
\hline Outros países & 2 & 12,5 \\
\hline Total & 8 & 64 \\
\hline
\end{tabular}

A tabela 5 apresenta os países que mais publicam, entre os artigos selecionados, considerando os autores presentes em todos os artigos, sem exclusão de autores repetidos. Assim, pode-se observar que a produção científica acerca das Born Globals tem recebido atenção em diversas áreas do globo, sendo distribuídos, para a amostra de artigos mais relevantes para o tema, em 19 países. A tabela 6 apresenta os autores que mais publicaram sobre planejamento estratégico, de acordo com o procedimento bibliométrico aplicado.

Tabela 6: Autores que mais publicaram sobre o tema.

\begin{tabular}{|l|l|l|}
\hline \multicolumn{1}{|c|}{ Autor } & \multicolumn{1}{c|}{ Número de Artigos } & \multicolumn{1}{c|}{ \% de artigos } \\
\hline FREEMAN, S. & $4 \quad 13,33$ \\
\hline KNIGHT, G. A. & 3 & 10 \\
\hline RIALP-CRIADO, A. & 2 & 6,67 \\
\hline CAVUSGIL, S. T. & 2 & 6,67 \\
\hline EFRAT, K. & 2 & 6,67 \\
\hline LIESCH, P. W. & 2 & 6,67 \\
\hline SHOHAM, A. & 2 & 6,67 \\
\hline
\end{tabular}

A tabela 6 apresenta os autores mais frequentes dos 30 artigos selecionados. Vale destacar que a autora que publicou mais artigos foi o Susan Freeman, sendo primeira autora de 3 artigos; seguida por Gary A. Knight, primeiro autor de 2 artigos; Kalanit Efrat, primeiro autor de 2 artigos e Alex Rialp-Criado, sendo primeiro autor de 1 artigo. Embora S. Tamer Cavusgil, Peter W. Liesch, e Aviv Shoham sejam autores de 2 artigos, eles não foram primeiros autores nenhuma vez.

Susan Freeman é PhD. em marketing, sendo professora de negócios internacionais na Universidade do Sul da Austrália, Austrália. A autora possui mais de 47 artigos científicos publicados e cerca de 657 citações. Gary A Knight é PhD. e professor de negócios globais na Universidade de Willamette, Estados Unidos da América. O autor possui mais de 14 artigos científicos e cerca de 2445 citações. Alex Rialp-Criado é PhD. e professor de Business Economics na Universidade Autônoma de Barcelona, Espanha. O autor possui mais de 62 artigos científicos publicados e mais de 1049 citações.

S. Tamer Cavusgil é PhD. e professor de negócios na Universidade do Estado da Georgia, Estados Unidos da América. $O$ autor possui mais de 205 artigos científicos publicados, com cerca de 10621 citações. Kalanit Efrat é PhD. e diretora do programa de marketing do Ruppin Academic Center, Israel. A autora possui mais de 9 artigos publicados e cerca de 43 citações. Peter W. Liesch é PhD. em economia e professor de negócios internacionais na Universidade de Queensland, Austrália. O autor possui mais de 146 artigos científicos publicados e cerca de 1393 citações. Aviv Shoham é PhD. e diretor do departamento de administração de negócios na Universidade de Haifa, Israel. O autor possui mais de 121 artigos científicos publicados e cerca de 2585 citações. 


\section{CONSIDERAÇÕES FINAIS}

Com o objetivo de analisar o estado da arte da produção científica internacional sobre Born Globals, os autores realizaram uma análise bibliométrica em três bases de dados internacionais, a EBSCO, a Science Direct e a Web of Science. A análise bibliométrica permitiu selecionar 30 artigos, considerados os mais importantes na área de Born Globals.

O ano de 2014 foi o ano com maior número de publicações, somando um total de $23,33 \%$ dos artigos selecionados, ou 7 artigos. $\mathrm{O}$ artigo com maior número de citações é intitulado 'Innovation, organizational capabilities, and the born-global firm', publicado em 2004, correspondendo a $46 \%$ das citações, ou 1756, em número absoluto.

Entre os artigos selecionados, as palavras-chave que mais se repetiram foram: Born Global, Internationalization, International Enterpreneurship e Small and medium enterprises (SMEs). Essas palavras são justificadas pelo objetivo do bibliométrico em identificar os artigos mais relevantes para o tema de Born Globals, estando alinhadas ao tema.

Faz-se necessário também elucidar que 6 revistas científicas são responsáveis por mais de 63,33\% dos artigos responsáveis, ou 19 artigos. Essas revistas são: Journal of World Business, Management International Review, Journal of International Entrepreneurship, Journal of International Business Studies, Journal of International Marketing, e European Journal of International Management. A ampla distribuição geográfica dos autores indica a crescente relevância do tema, Born Globals, em diversas partes do mundo.

\section{REFERÊNCIAS}

ALMOR, T.; TARBA, S. Y.; MARGALIT, A.. Maturing, Technology-Based, Born-Global Companies: Surviving Through Mergers and Acquisitions. Management International Review, v.54, n.4, p.421-444, 2014. DOI: http://doi.org/10.1007/s11575-014-0212-9

ANDERSEN, O.; BUVIK, A.. Firms internationalization and alternative approaches to the international customer/market selection. International business review, v.11, n.3, p.347-363, 2002. DOI:

http://doi.org/10.1016/S0969-5931(01)00064-6

APPOLINÁRIO, F.. Metodologia da ciência: filosofia e prática da pesquisa. São Paulo: Learning, 2009.

ASPFORS, J.; FRANSSON, G.. Research on mentor education for mentors of newly qualified teachers: a qualitative metasynthesis. Teaching and Teacher Education, v.48, p.75-86, 2015.

ASSIS, M. C.. Metodologia do trabalho científico. João Pessoa: UFPB, 2014

BARONCHELLI, G.; CASSIA, F.. Exploring the antecedents of born-global companies international development. International Entrepreneurship and Management Journal, v.10, n.1, p.67-79, 2014. DOI: http://doi.org/10.1007/s11365-011-0197-9
CANCINO, C. A.. Rapid Internationalization of SMEs: Evidence from Born Global Firms in Chile. Innovar: Revista de Ciencias Administrativas y Sociales, v.24, n.54, p.141-151, 2014.

CASAS, R.; VILMA, D.. The impact of external business environment factors to internationalization of 'Born Global' Companies By Promoting Entrepreneurship.

Transformations In Business \& Economics, v.90, n.3, p.120 135, 2011.

CASTRO, A. A.. Revisão Sistemática e Meta-análise. São Paulo: 2001

CRICK, D.. The internationalisation of born global and international new venture SMEs. International Marketing Review, v.26, n.5, p.453-476, 2009. http://doi.org/10.1108/02651330910971986

DIB, L. A.; ROCHA, A.; SILVA, J. F.. The internationalization process of Brazilian software firms and the born global phenomenon: Examining firm, network, and entrepreneur variables. Journal Of International Entrepreneurship, v.8, n.3, p.233-253, 2010. http://doi.org/10.1007/s10843-0100044-z

EFRAT, K.; SHOHAM, A.. Born global firms: The differences between their short- and long-term performance drivers. Journal Of World Business, v.47, n.4, p.675-685, 2012. DOI: http://doi.org/10.1016/j.jwb.2012.01.015 
EFRAT, K.; SHOHAM, A.. Environmental characteristics and technological capabilities' interaction in high-technology born global firms. European Journal of International Management, v.5, n.3, p.271-284, 2011. DOI: http://doi.org/10.1504/ejim.2011.039942

ERLINGSSON, C.; BRYSIEWICZ, P.. Orientation among multiple truths: An introduction to qualitative research. African Federation for Emergency Medicine, v.3, p.92-99, 2013.

FAN, T.; PHAN, P.. International new ventures: revisiting the influences behind the 'born-global' firm. Journal of International Business Studies, v.38, n.7, p.1113-1131, 2007.

FORREST, J.. Strategic Alliances and the Small Technologybased Firm. Journal of Small Business Management, v.28, n.3, p.37-45, 1990.

FREEMAN, S.. A model of rapid knowledge development: The smaller born-global firm. International Business Review, v.19, n.1, p.70-84, 2010. DOI:

http://doi.org/10.1016/j.ibusrev.2009.09.004

FREEMAN, S.; CAVUSGIL, S. T.. Toward a Typology of Commitment States Among Managers of Born-Global Firms: A Study of Accelerated Internationalization. Journal Of International Marketing, v.15, n.4, p.1-40, 2007. DOI: http://doi.org/10.1509/jimk.15.4.1

FREEMAN, S.; DELIGONUL, S.; CAVUSGIL, T.. Strategic Restructuring by Born-globals Using Outward and InwardOriented Activity. International Marketing Review, v.30, n.2, p.156-182, 2013. DOI:

http://doi.org/10.1108/02651331311314574

FREEMAN, S.; EDWARDS, R.; SCHRODER, B.. How Smaller Born-Global Firms Use Networks and Alliances to Overcome Constraints to Rapid Internationalization. Journal of International Marketing, v.14, n.3, p.33-63, 2006.

FREEMAN, S.; HUTCHINGS, K.; CHETTY, S.. Born-globals and Culturally Proximate Markets. Management International Review, v.52, n.3, p.425-460, 2012. DOI: http://doi.org/10.1007/s11575-011-0109-9

GABRIELSSON, M.. Branding strategies of born globals. Journal of International Entrepreneurship, v.3, n.3, p.199-222, 2005. DOI: http://doi.org/10.1007/s10843-0050401-5

GERSCHEWSKI, S.; ROSE, E. L.; LINDSAY, V. J.. Understanding the drivers of international performance for born global firms: An integrated perspective. Journal Of World Business, v.50, n.3, p.558-575, 2015. DOI:

http://doi.org/10.1016/j.jwb.2014.09.001

GRANITSKY, J.. Strategies for innate and adoptive exporters: Lessons from Israel's case. In: International Marketing

Review, v.6, n.5, p.50-65, 1989. DOI:

http://doi.org/10.1108/EUM0000000001523

HAGEN, B.; ZUCCHELLA, A.. Born Global or Born to Run? The Long-Term Growth of Born Global Firms. Management International Review, v.54, n.4, p.497-525, 2014. DOI: http://doi.org/10.1007/s11575-014-0214-7
HARVESTON, P. D.; KEDIA, B. L.; DAVIS, P. S. Internationalization of born global and gradual globalizing firms: The impact of the manager. Journal of Competitiveness Studies, v.8, n.1, p.92, 2000.

HASHAl, N.. Sequencing the expansion of geographic scope and foreign operations by 'born global' firms. Journal of International Business Studies, v.42, n.8, p.995-1015, 2011. http://doi.org/10.1057/jibs.2011.31

HEMAIS, C. A.; HILAL, A.. Teorias, paradigma e tendências em negócios internacionais: de Hymer ao empreendedorismo. Rio de Janeiro: Mauad, 2004.

HOLTBRUGGE, D.; WESSELY, B.. Initiating forces and success factors of born global firms. European Journal of International Management, v.3, n.2, p.232-260, 2009. DOI: http://doi.org/10.1504/ejim.2009.024324

IETTO-GILLIES, G.. Transnational corporations:

Fragmentation amidst integration. Abingdon: Routledge, 2002.

JOHANSON, J.; MATTSSON, L. G.. Internationalisation in industrial systems: a network approach. In: HOOD, N. Y. V. J.. Strategies en Global Competition. Dover: Crom Helm, 1988. DOI: http://dx.doi.org/10.1057/9781137508829 5

JOHANSON, J.; VAHLNE, J. E.. The mechanism of internationalisation. International marketing review, v.7, n.4, 1990. http://doi.org/10.1108/02651339010137414

JOHANSON, J.; VAHLNE, J. E.. The Uppsala

internationalization process model revisited: From liability of foreignness to liability of outsidership. Journal of international business studies, v.40, n.9, p.1411-1431, 2009. DOI: http://doi.org/10.1057/jibs.2009.24

JOHANSON, J.; WIEDERSHEIM-PAUL, F.. The internationalization of the firm: four swedish cases 1. Journal of management studies, v.12, n.3, p.305-323, 1975.

JOLLY, V. K.; ALAHUHTA, M.; JEANNET, J. P.. Challenging the incumbents: How high technology start-ups compete globally. Strategic Change, v.1, n.2, p.71-82, 1992.

KARAGOZOGLU, N.; LINDELL, M.. Internationalization of small and medium-sized technology-based firms: An exploratory study. Journal of small business management, v.36, n.1, p.44, 1998.

KAUR, S.; SANDHU, M. S.. Internationalisation of born global firms: Evidence from Malaysia. Journal Of The Asia Pacific Economy, v.19, n.1, p.101-136, 2013. DOI: http://doi.org/10.1080/13547860.2013.818426

KNIGHT, G. A.; CASVUGIL, S. T.. The born global firm: A challenge to traditional internationalization theory. In: CAVUSGIL, S. T.; MADSEN, T.. Advances in international marketing, Greenwich, v.8, p.11-6, 1996.

KNIGHT, G. A.; CAVUSGIL, S.. Tamar: Innovation, organizational capabilities, and the born-global firm. Journal of International Business Studies, v.35, n.2, p.124-141, 2004.

KNIGHT, G. A.; LIESCH, P. W.. Internationalization: From incremental to born global. Journal Of World Business, v.51, 
n.1, p.93-102, 2016. DOI:

http://doi.org/10.1016/j.jwb.2015.08.011

LAANTI, R.; GABRIELSSON, M.; GABRIELSSON, P.. The globalization strategies of business-to-business born global firms in the wireless technology industry. Industrial Marketing Management, v.36, n.8, p.1104-1117, 2007. DOI: http://doi.org/10.1016/i.indmarman.2006.10.003

MADSEN, T. K.. Early and rapidly internationalizing ventures: Similarities and differences between classifications based on the original international new venture and born global literatures. Journal Of International Entrepreneurship, v.11, n.1, p.65-79, 2012. DOI: http://doi.org/10.1007/s10843-012$\underline{\text { 0099-0 }}$

MADSEN, T. K.; SERVAIS, P.. The internationalization of born globals: an evolutionary process?. International business review, v.6, n.6, p.561-583, 1997. DOI: http://doi.org/10.1016/S0969-5931(97)00032-2

MARCONI, M.; LAKATOS, E. M.. Fundamentos de Metodologia Científica. 5 ed. São Paulo: Editora Atlas, 2003.

MOEN, O.. The born globals: a new generation of small European exporters. International Marketing Review, v.19 n.2, p.156-175, 2002. DOI: http://doi.org/10.1108/02651330210425015

MONFERRER, D.; BLESA, A.; RIPOLLÉS, M.. Born globals trough knowledge-based dynamic capabilities and network market orientation. Business Research Quarterly, v.18, n.1, p.18-36, 2015. DOI:

http://doi.org/10.1016/i.brq.2014.04.001

NEUMANN, R. W.; HEMAIS, C. A.. Produção internacional e comportamento organizacional no processo de internacionalização: podem as teorias explicar o comércio internacional?. In: HEMAIS, C. A.. O Desafio dos Mercados Externos: teoria e prática na internacionalização da firma. Mauad: 2005.

NORDMAN, E. R.; MELÉN, S.. The impact of different kinds of knowledge for the internationalization process of born globals in the biotech business. Journal of World Business, v.43, n.2, p.171-185, 2008. DOI:

http://doi.org/10.1016/i.jwb.2007.11.014

NORDSTRÖM, K.; VAHLNE, J.. Is the globe shrinking? Psychic distance and the establishment of Swedish sales subsidiaries during the last 100 years. In: SILVA, M; ROCHA, A; FIGUEIREDO, O. Medindo o construto da distância psíquica. In: INTERNATIONAL TRADE AND FINANCE ASSOCIATION'S ANNUAL CONFERENCE. Anais. Laredo: 1992.

NUMMELA, N.. Strategic Decision-Making of a Born Global: a comparative study from three small open economies. Management International Review, v.54, n.4, p.527-550, 2014. DOI: http://doi.org/10.1007/s11575-014$\underline{0211-x}$

OVIATT, B. M.; MCDOUGALL, P. P.. Toward a theory of international new ventures. Journal of international business studies, v.25, n.1, p.45-64, 1994. DOI: http://doi.org/10.1057/palgrave.jibs.8490193

PRODANOV, C. C.; FREITAS, E. C.. Metodologia do trabalho científico: Métodos e Técnicas da Pesquisa e do Trabalho
Acadêmico. 2 ed. Nova Hamburgo: Universidade Feevale, 2013.

RENNIE, M. W.. Born global. The McKinsey Quarterly, n.4, p.45-53, 1993.

RIALP-CRIADO, A.. The born-global phenomenon: A comparative case study research. Journal of International Entrepreneurship, v.3, n.2, p.133-171, 2005. DOI: http://doi.org/10.1007/s10843-005-4202-7

RIALP-CRIADO, A.; GALVÁN-SÁNCHEZ, I.; SUÁREZ-ORTEGA, S. M.. A configuration-holistic approach to born-global firms' strategy formation process. European Management Journal, v.28, n.2, p.108-123, 2010. DOI:

http://doi.org/10.1016/j.emj.2009.05.001

SILVA, M. C.. Procedimentos metodológicos para a elaboração de projetos de pesquisa relacionados a dissertações de mestrado em Ciências Contábeis. Revista Contabilidade \& Finanças, São Paulo, p.97-104. 2004.

TANURE, B.; CYRINO, Á. B.; PENIDO, É. Estratégias de internacionalização: evidências e reflexões sobre as empresas brasileiras. In: FLEURY, A.; FLEURY, M. T. L.. Internacionalização e os Países Emergentes. São Paulo: Atlas, 2007.

TAYLOR, M.; JACK, R. Understanding the pace, scale and pattern of firm internationalization: An extension of the 'born global' concept. International Small Business Journal, v.31, n.6, p.701-721, 2012. DOI: http://doi.org/10.1177/0266242611431992

TEIXEIRA, S.; DIZ, H.. Estratégias de internacionalização. Lisboa: Publisher Team, 2005.

THAI, M. T. T.; CHONG, L. C.. Born-global: The case of four Vietnamese SMEs. Journal of International Entrepreneurship, v.6, n.2, p.72-100, 2008. DOI: http://doi.org/10.1007/s10843-008-0021-y

TORRES-ORTEGA, R.. How to measure born-global firms' orientation towards international markets?. Revista Española de Investigación En Marketing Esic, Amsterdã, v.19, n.2, p.107-123, 2015.

DOI: http://doi.org/10.1016/j.reimke.2015.04.001

TR. Thomson Reuters. Web of Science. Eagan: TR, 2015.

TRUDGEN, R.; FREEMAN, S.. Measuring the Performance of Born-Global Firms Throughout Their Development Process: The Roles of Initial Market Selection and Internationalisation Speed. Management International Review, v.54, n.4, p.551579, 2014. DOI: http://doi.org/10.1007/s11575-014-0210-y

UTL. University of Texas Library. Business Source Complete. Austin: UTL, 2015.

WEERAWARDENA, J.. Conceptualizing accelerated internationalization in the born global firm: A dynamic capabilities perspective. Journal Of World Business, v.42, n.3, p.294-306, 2007. DOI:

http://doi.org/10.1016/j.jwb.2007.04.004

WEISFELDER, C. J.. Internationalization and the multinational enterprise: development of a research tradition. In: AXINN, C. N.; MATTHYSSENS, P.. Reassesing the Internationalization of the Firm. Bingley: Emerald Group Publishing Limited, 
2001. p.13-46. DOI: http://doi.org/10.1016/S14747979(01)11015-X

WIEDERSHEIM-PAUL, F.; OLSON, H. C.; WELCH, L. S.. Preexport activity: The first step in internationalization. Journal of International Business Studies, v.9, n.1, p.47-58, 1978. DOI: http://doi.org/10.1057/palgrave.jibs. 8490650

ZHANG, J.; DAl, X.. Research on Chinese Born global firms' international entrepreneurial mechanism and development mode. International Business and Management, v.7, n.1, p.77-83, 2013. DOI:

http://doi.org/10.3968/j.ibm.1923842820130701.1125

ZHANG, M.; TANSUHAJ, P.; MCCULLOUGH, J.. International entrepreneurial capability: the measurement and a comparison between born global firms and traditional exporters in China. Journal Of International Entrepreneurship, v.7, n.4, p.292-322, 2009. DOI: http://doi.org/10.1007/s10843-009-0042-1 